\title{
A Research on the Purchase Quantity Allocation between Contract Market and Power Auction Market
}

\author{
Haihuan Wang ${ }^{1}$ Bin Zou $^{2}$ \\ ${ }^{1}$ School of Communication and Control Engineering, Jiangnan University, Wuxi 214122, China \\ ${ }^{2}$ Control Science and Engineering Research Center, Jiangnan University, Wuxi 214122, China
}

\begin{abstract}
In open electricity market, electricity purchasers will trade in two different ways of marketing. Contract and power auction market, a typical decisionmaking is how to determine purchasing proportion of two markets to reduce purchase expenses and avoid risks. Based on chance-constrained programming, a new purchase decision-making optimization model with stochastic constraint of Distribution Company is simplified into definite model. Solution is genetic algorithm based on stochastic simulation which coordinates purchase expenses and risks successfully. Finally, a numerical simulation demonstrates optimal distribution strategy clearly. Results indicate that it's helpful for electricity purchasers to seek optimal investment strategy in electricity market.
\end{abstract}

Keywords: Electricity market, Electricity distribution, Chance-constrained programming, Genetic algorithm, Risk analysis

\section{Introduction}

There is variety of different electricity trading forms in electricity market. The most significant one is the form of contract market and the auction market. Trading quantities and trading prices are established by the government's coordination in principle during the contract transaction. However, the auction market bids according to the market clearing in principle. The market members may obtain the most effective transaction project using the different combination of transaction ways [6][7]. Speaking of the sole customer's market, a typical decision-making issue is how to determine the purchasing proportion of the two markets, contract market and auction market.

In the electricity purchaser's risk management of purchasing decision-making, a purchase distrib- ution research [8]-[9] on the three order markets which are day-ahead market, hour-ahead market and real time market has been done, it discussed the description and impact of risks. Guo Jin and Tan Zhongfu [10] have established a target model of profit maximization according to Markowitz theory of the risk investment, variance of profit was considered as an index of measuring risk. Introduction of (the power company's attention to risk) converted a double- objective programming into a single one, therefore, the value of determines the purchase of electricity distribution on a large extent, so the key of this model application is to evaluate correctly. Chi-Keung Woo [11] assessed the risk of purchase cost through cost exposure and VAR which combine the anticipated loss size in future with the possibility of it occurs, it lets the distribution company not only know the loss scale but also know the possibility of it occurs. In [12], a Monte Carlo simulation was used to seek the optimal solution of the contract allocation, convergence rate of Monte Carlo has nothing to do with the problem's dimension, moreover Monte Carlo understands easily, it is easy to code, but there is still weakness, its computation load is extremely big, if you wants to increase a digit precision, you need to increase 100 times of computation loads. Furthermore its convergence rate is slow.

This paper aimed at the in-depth study of the purchasing allocation issue and risk assessment methodologies in the power market. By analyzing the electricity purchasers' purchasing features, we take advantage of chance constrained programming which can depict risk issue naturally to denote the quantity allocation issue of the two trading markets with a chance constrained programming model. A definite solvable model can be obtained through the model transformation. This paper also further discusses the mutual influencing relations of the contract market and the spot market by using this model. 


\section{Mathematical model}

\subsection{Characteristic of contract market and spot market}

Our model establishment is under such market rules: Purchaser can purchase its needed electricity quantity from the contract market and the spot market (power auction market). The purchasing quantity and purchasing price are determined through the two sides' coordination in the contract market. However, the power auction market's purchasing quantity and purchasing price are determined by the auction market rules, here according to the market clearing rules.

Any of market members can't control the market' price, moreover there is great fluctuation of the present market price, so it leads the contract purchase and the proportion to be the important content of avoiding risks. When considering the distribution company's expense and risk, we should consider both the demand uncertainty and the price and risk of the contract market and spot market. Because any of market participants can't control the market completely, the prices for the two markets are all in random variables, and they are relevant [10].

According to the analysis to power auction market in [13], price of power auction market is a random variable following the normal distribution which is in condition to load. We can confirm its basis distribution of price when a future time load has been forecasted. Through the detailed reasoning in [14], we know that price of power auction market follows Logarithmic normal distribution, namely the logarithm of the actual price will follows the normal distribution. Given that $\lambda_{2}$ is the price of power auction market, and $\lambda_{2} \sim N\left(\mu, \sigma^{2}\right)$, so $\ln \lambda_{2} \sim N\left(\mu_{2}, \sigma_{2}^{2}\right)$. Then the price probability density function of power auction market based on the forecast load is as follows:

$$
f\left(\lambda_{2} \mid L\right)= \begin{cases}\frac{1}{\sqrt{2 \pi} \sigma \lambda_{2}} \epsilon^{-\frac{(l n \lambda-\mu)^{2}}{2 \sigma^{2}}} & \text { if } \lambda_{2}>0 \\ 0 & \text { if } \quad \lambda_{2}<0\end{cases}
$$

$\lambda_{2}$ denotes a random variable of the power auction market's price; $L$ denotes load demand quantity; $\mu$ and $\sigma^{2}$ is called the logarithmic mean and logarithmic variance of Logarithmic normal distribution. Then the purchase expense of the distribution company in the power auction market can be represented as:

$$
R_{s}=\lambda_{2} \times Q_{s}
$$

$Q_{s}$ is the purchase quantity in power auction market; The contract market's results are obtained from the bilateral coordination. According to the beforehand experience, trading price can't been determined during the quantity allocation. We suppose $\lambda_{1}$ to be the contract price and $\lambda_{1} \sim$ $N\left(\mu_{1}, \sigma_{1}^{2}\right)$, the purchase expense of contract market is given as:

$$
R_{c}=\lambda_{1} \times Q_{c}
$$

$\lambda_{1}$ denotes a random variable of contract price which subjects to normal distribution. $Q_{c}$ is the trading quantity of contract market.

Given that is the total demand quantity of distribution company in a future time, it is obvious that:

$$
Q=Q_{s}+Q_{c}
$$

\subsection{Allocation model of pur- chasing quantity}

The purchase allocation model's goal of the distribution company is to minimize the purchase expense, namely the following model:

$$
\begin{array}{ll}
\min _{q} & f=q \cdot \lambda_{1}+(Q-q) \cdot \lambda_{2} \\
\text { s.t. } & 0 \leq q \leq Q
\end{array}
$$

$\lambda_{1}$ denotes a random variable of contract market price; $\lambda_{2}$ is power auction market's price, a random variable; $Q$ is the distribution company's total quantity, a certain value; $q$ denotes the purchase quantity of contract market, decision variable of the model.

Because the both price are all random variables, purchase expense is also a random variable, this kind model can't be solved. The usual method is to turn the objective function to the minimum its expectation. Then this article structures further a stochastic constraint programming. Concrete model is given as follows:

$$
\begin{array}{ll}
\min _{q} & \bar{f} \\
\text { s.t. } & \quad \operatorname{Pr}\{f \leq \bar{f}\} \geq \beta \\
& 0 \leq q \leq Q
\end{array}
$$

$f$ is random variable which denotes the purchase expense in formula $(5) ; \bar{f}$ is a certain value whose meaning is an appointed level of purchase expense; $\beta$ is an appointed probability which denotes that purchase expense is no bigger than the appointed confidence level. Other Symbols are similar with formers. 
The meaning of above model is to choose the contract purchasing quantity, so it can satisfy that realize probability meets the required confidence level when the purchasing expense is the minimum. It is a typical chance constrained model.

\section{Solution to the model}

\subsection{The transformation based on chance constrained pro- gramming model}

According to [18], model (6) can be transformed to the following model:

$$
\begin{gathered}
\min _{q} \overline{\bar{f}} \\
\text { s.t. }\left\{\begin{array}{c}
\bar{f} \geq \sup \left\{K \mid K=F_{f}^{-1}(\beta)\right\} \\
0 \leq q \leq Q
\end{array}\right.
\end{gathered}
$$

$F_{f}$ is the distribution function of $f=q \cdot \lambda_{1}+(Q-$ $q) \cdot \lambda_{2}$. From the detailed reasoning in [14], we can know that:

$$
\mu_{2}=\epsilon^{\mu+\frac{1}{2} \sigma^{2}}, \sigma_{2}^{2}=\epsilon^{2 \mu+2 \sigma^{2}}-\epsilon^{2 \mu+\sigma^{2}}
$$

Symbol nomenclature see front explain. So we can deduce that $f \sim N\left(\bar{\mu}, \bar{\sigma}^{2}\right)$, and its mean and variance are given as follows:

$$
\begin{gathered}
\bar{\mu}=q \mu_{1}+(Q-q) \mu_{2} \\
\bar{\sigma}^{2}=q^{2} \sigma_{1}^{2}+(Q-q)^{2} \sigma_{2}^{2} \\
+2 \rho q \sigma_{1} \sigma_{2}(Q-q)
\end{gathered}
$$

Model (7) may further simplify for the standard normal distribution form:

$$
\begin{aligned}
& \min _{q} \overline{\bar{f}} \\
& \text { s.t. }\left\{\begin{array}{c}
\bar{f} \geq \bar{u}+\bar{\sigma} \Phi^{-1}(\beta) \\
0 \leq q \leq Q
\end{array}\right.
\end{aligned}
$$

$\Phi$ is the standard normal distribution function. The model (7) is a definite model. But then the relation of $F_{f}$ and $q$ is nonlinear, so the model is a typical nonlinear constraint optimal issue. To this kind of optimized problem, genetic algorithm is an excellent solution.

\subsection{Genetic algorithm solves objective function}

Genetic algorithm's Specific flow is shown in Fig.1.

The selection of codes adopts binary system symbols with length $l=10$ to denote the quantity $\left(q_{1}, q_{2}\right)$ of spot market and contract market.

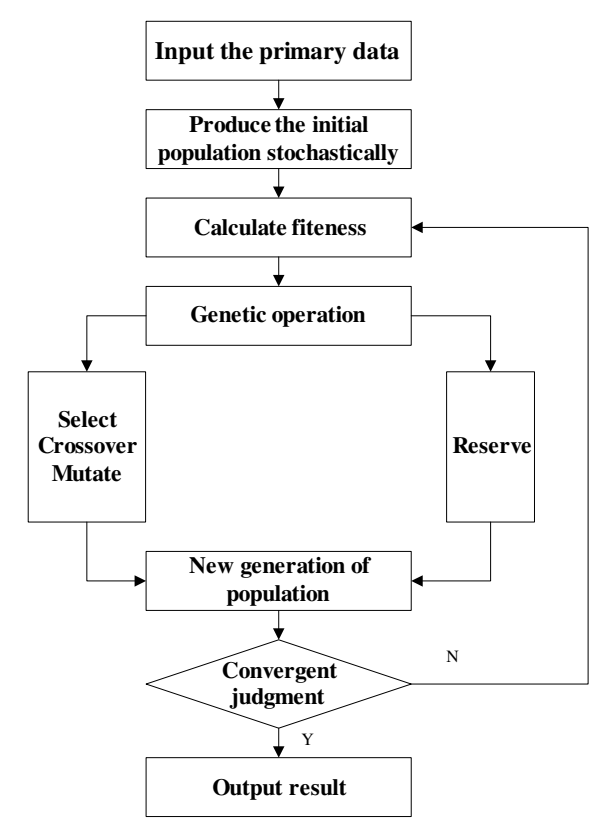

Fig. 1: Genetic algorithm's flow chart.

The entire chromosome expresses to be $\left[q_{1} \mid q_{2}\right]$, chromosome's length is 20, the former 10 denotes $q_{1}$, and the latter 10 denotes $q_{2}$.

We adopts the fitness function which is minimum objective function issue, $C_{\max }$ is a fixed value, in order to guarantee the fitness's non-negative, it must be big enough. When the total needed quantity is determinated and also history price is known, total purchasing expense can be forecasted easily, so it is still easy to determine $C_{\max }$, we assume that $C_{\max }=10000000 \$$ in present paper.

$$
\operatorname{Fitness}(x)=\left\{\begin{array}{cl}
C_{\max }-f(x) & f(x)<C_{\max } \\
0 & f(x) \geq C_{\max }
\end{array}\right.
$$

Substitute the minimum objective function issue in model (7) for the in model (11), we suppose $f_{t}=$ $\bar{u}+\bar{\sigma} \Phi^{-1}(\beta)$, objective function model of genetic algorithm is given as:

$$
\begin{array}{ll}
\min _{q} & \text { Fitness }(q)=\left\{\begin{array}{cl}
C_{\max }-f_{t} & f_{t}<C_{\max } \\
0 & f_{t} \geq C_{\max }
\end{array}\right. \\
\text { st } & 0 \leq q \leq Q
\end{array}
$$

Decision variable is $q$.

\section{Example analysis}

On the basis of a certain area distribution company's analysis of historical data ${ }^{[17]}$, the price distribution, correlation coefficient and load forecast- 
ing of the contract market and the spot market are estimated as shown in tab.1.

\begin{tabular}{|l|l|l|}
\hline & $\begin{array}{l}\text { Symbol } \\
\text { express }\end{array}$ & Distribution \\
\hline Contract price & $\lambda_{1}$ & $\mathrm{~N}(55,189)$ \\
\hline Spot price & $\ln \lambda_{2}$ & $\mathrm{~N}(72,1122)$ \\
\hline Load forecasting & $Q$ & $\mathrm{~N}(1800,2803)$ \\
\hline correlation coefficient & $\rho$ & 0.1 \\
\hline Unit: $\lambda_{1}-M w h, \ln \lambda-M w h, Q-M w h$ \\
\hline
\end{tabular}

Table 1: Statistical Features of Variable.

Changes the objective function's confidence level $\beta=0.95,0.90,0.85,0.80$, we can obtain the contract purchase quantity $q$, spot purchase quantity $Q-q$, purchasing allocation proportion $\gamma=$ $q / Q$ and the expected value of purchase expense $\bar{\mu}$ as shown in tab. 2 .

\begin{tabular}{|l|l|l|l|l|}
\hline$\beta$ & 0.99 & 0.95 & 0.90 & 0.85 \\
\hline$q$ & 1733.4 & 1785.6 & 1798.2 & 1798.2 \\
\hline$Q-q$ & 66.6 & 14.4 & 1.8 & 1.8 \\
\hline$\gamma$ & 0.963 & 0.992 & 0.999 & 0.999 \\
\hline $\bar{u}$ & 100132 & 99244 & 99030 & 99030 \\
\hline$\Phi^{-1}(\beta)$ & 2.33 & 1.745 & 1.28 & 1.14 \\
\hline \multicolumn{4}{|l|}{ Unit:q $\sim$ Mwh, $\sim \sim M w h, \bar{u} \sim \$ / M w h$} \\
\hline
\end{tabular}

Table 2: Changes Produced by Different $\beta$.

From tab.2, we may see model's final result, when $\beta=0.90, q$ is $1798.2 M w h, Q-q$ is $1.8 M w h$, $\gamma$ is 0.999 , namely distribution company will choose the relative stable contract market.

In order to narrate the influence of two markets' mean value and standard variance to purchasing allocation strategy better, we change $\mu_{1}, \mu_{2}, \sigma_{1}, \sigma_{2}$ to analyze the purchasing strategy's variety conditions when $\beta=0.95$ and others keeps invariable.

First, change the standard variance $\sigma_{1}$ and $\sigma_{2}$ of two markets, the results are given as tab. 3 and tab. 4.

\begin{tabular}{|l|l|l|l|l|}
\hline \multicolumn{5}{|l|}{ Change $\sigma_{1}$ when $\beta=0.95$} \\
\hline$\sigma_{1}$ & $q$ & $Q-q$ & $\gamma$ & $\bar{u}$ \\
\hline 11 & 1798.2 & 1.8 & 0.999 & 99030 \\
\hline 13 & 1798.2 & 1.8 & 0.999 & 99030 \\
\hline 15 & 1755 & 45 & 0.975 & 99765 \\
\hline 17 & 1699.2 & 100.8 & 0.944 & 100713 \\
\hline 19 & 1636.2 & 163.8 & 0.909 & 101784 \\
\hline Unit: $q \sim M w h, Q-q \sim M w h, \bar{u} \sim \$ / M w h$ \\
\hline
\end{tabular}

Table 3: Changes Produced by Different $\sigma_{1}$.

\begin{tabular}{|l|l|l|l|l|}
\hline Change $\sigma_{2}$ when $\beta=0.95$ \\
\hline$\sigma_{2}$ & $q$ & $Q-q$ & $\gamma$ & $\bar{u}$ \\
\hline 33 & 1783.8 & 16.2 & 0.991 & 99275 \\
\hline 35 & 1789.2 & 10.8 & 0.994 & 99183 \\
\hline 37 & 1794.6 & 5.4 & 0.997 & 99091 \\
\hline 39 & 1798.2 & 1.8 & 0.999 & 99030 \\
\hline 41 & 1798.2 & 1.8 & 0.999 & 99030 \\
\hline Unit: $q \sim M w h, Q-q \sim M w h, \bar{u} \sim \$ / M w h$ \\
\hline
\end{tabular}

Table 4: Changes Produced by Different $\sigma_{2}$.

When the contract market's standard variance $\sigma_{1}$ reduces, then the purchasing quantity of contract market increases, but the most is $1798.2 \mathrm{Mwh}$, and the purchasing quantity of spot market reduces, purchasing allocation proportion ascends, total expected purchasing expense reduces. When $\sigma_{1}=15$, purchasing quantity in the contract market is $1755 \mathrm{Mwh}$, in the spot market is $45 \mathrm{Mwh}$, and $\gamma=0.975$. The variety results of $\sigma_{2}$ can be acquired likewise. This conclusion illuminates that when a market standard variance increases, it represents its market risk increasing, then purchaser will reduce the purchase quantity in this market, meanwhile increase another market's purchase quantity. When increases purchase quantity in the spot market, it will increase the total expected purchase expense.

Well then, change the standard mean value $\mu_{1}$ and $\mu_{2}$ of the contract market and the spot market, the results are given as tab. 5 and tab.6:

\begin{tabular}{|l|l|l|l|l|}
\hline \multicolumn{5}{|l|}{ Change $\mu_{1}$ when $\beta=0.95$} \\
\hline$\mu_{1}$ & $q$ & $Q-q$ & $\gamma$ & $\bar{u}$ \\
\hline 51 & 1798.2 & 1.8 & 0.999 & 91837 \\
\hline 53 & 1798.2 & 1.8 & 0.999 & 95434 \\
\hline 55 & 1785.6 & 14.4 & 0.992 & 99244 \\
\hline 57 & 1760.4 & 39.6 & 0.978 & 103194 \\
\hline 59 & 1737 & 63 & 0.965 & 107019 \\
\hline Unit: $q \sim M w h, Q-q \sim M w h, \bar{u} \sim \$ / M w h$ \\
\hline
\end{tabular}

Table 5: Changes Produced by Different $\mu_{1}$.

\begin{tabular}{|l|l|l|l|l|}
\hline \multicolumn{5}{|l|}{ Change $\mu_{2}$ when $\beta=0.95$} \\
\hline$\mu_{2}$ & $q$ & $Q-q$ & $\gamma$ & $\bar{u}$ \\
\hline 68 & 1737 & 63 & 0.965 & 99819 \\
\hline 70 & 1760.4 & 39.6 & 0.978 & 99594 \\
\hline 72 & 1785.6 & 14.4 & 0.992 & 99244 \\
\hline 74 & 1798.2 & 1.8 & 0.999 & 99034 \\
\hline 76 & 1798.2 & 1.8 & 0.999 & 99037 \\
\hline Unit: $q \sim M w h, Q-q \sim M w h, \bar{u} \sim \$ / M w h$ \\
\hline
\end{tabular}

Table 6: Changes Produced by Different $\mu_{2}$. 
When the spot market's price mean value $\mu_{2}$ increases, then the purchasing quantity in this market reduces, and the purchasing quantity of contract market increases, but the most is $1798.2 \mathrm{Mwh}$, purchasing allocation proportion ascends, total expected purchasing expense reduces.

When $\mu_{2}=70 \$ / M w h$, purchasing quantity in the contract market is $1760.4 M w h$, in the spot market is $39.6 \mathrm{Mwh}$, and $\gamma=0.978$. This conclusion illuminates that when a market's price increases, purchaser will reduce the purchase quantity in this market, meanwhile increase the other market's purchase quantity . When reduces purchase quantity in the spot market, and it will reduce the total expected purchase expense.

In summary, the enlargement of a market risk will lead to electricity purchaser choose another relative stable market; the ascension of a market price will lead to purchasing quantity of another market increases. This conclusion proves that there is relativity between the contract market and the spot market.

\section{Example analysis}

How to seek the optimal purchasing allocation quantity in power market for Distribution Company, therefore it can minimize the purchase expenses under certain risks. This article gives us the method which transforms the model to a definite optimal model with restraint and also gives the settlement algorithm based on the genetic algorithm. The actual data computation indicates that this method can provide consumers a purchasing quantity allocation decision-making under certain confidence level.

The further work will be how to enhance the efficiency of model's solution and consider all kinds of constraint conditions.

\section{Acknowledgement}

This work is partially supported by National Nature Science Foundation of China (Grant No.60574051).

\section{References}

[1] Hung-po Chao, Global Electricity Tranformation: The Critical Need for Integrated Market Design and Risk Management Research. Energy,31:923-939, 2006.
[2] S.J. Deng, S.S. Oren, Electricity Derivatives and Risk Management. Energy, 31:940-953, 2006.

[3] X.S. Ma, F.S. Wen, Y.X. Nia, J.X. Liu, Towards the Development of Risk Constrained Optimal Bidding Strategies for Generation Companies in Electricity Markets. Electric Power Systems Research, 73:305-312,2005.

[4] R.Dahlgen, C.C.Liu, J. Lawarree, Risk assessment in energy trading. IEEE PWS, 18: 507511, 2003.

[5] M. Liu, F.F.Wu, Managing Price Risk in a Multi-market Environment. IEEE PWS, 21: 1512-1519, 2006.

[6] X.L. Chen, Y.He, Y.H. Song, Study of Impacts of Physical Contracts and Financial Contracts on Bidding Strategies of GENCOs. Electrical Power and Energy Systems, 26: 715-723, 2004.

[7] E.Tanlapco, J.Lawarre, C.C.Liu, Hedging with Futures Contracts in a Deregulated Electricity Industry. IEEE PWS, 17: 577-582, 2006.

[8] Y.A. Liu, X.H. Guan, Market Allocation Problem for Energy Demander in Power Market. Proceedings of CSEE, 21: 20-23, 2001.

[9] Y.A. Liu, X.H. Guan, Optimization of Purchase Allocation in Dual Electric Power Markets with Risk Management. Automation of Electric System, 26: 41-44, 2002.

[10] J. Guo, W. Jiang, Z.F. Tan,Research on Optimized Power Purchasing of Power Suppliers under Risk Condition. Power System Technology, 28: 18-22, 2004.

[11] Chi-Keung Woo, Rouslan I. Karimov, Ira Horow- itz, Managing Electricity Procurement Cost and Risk by a Local Distribution Company. Energy Policy, 32: 635-645, 2004.

[12] I.Vehrilainen and J.Keppo, Managing Electricity Market Price Risk. Eur. J. Res,145:136147,2003

[13] Z.X. Zhu, B. Zou, The Risk Model of Distribution Utilities and Analyzing the Infection. Journal of Relay, 35: 56-60, 2007.

[14] Z.X. Zhu, B. Zou, Statistical Analysis of Dayahead Prices in PJM Market. Automation of Electric System, 30: 53-57, 2006.

[15] J. Wang, F.S. Wen, Rengang Yang, Optimal maintenance strategy for generation companies based on chance-constrained programming. Automation of Electric System, 28: 2731, 2004.

[16] Y.g. Li, S. Jiong, X.C. Li, A new bidding strategy for power plants Based on Chance-constrained Programming. Proceedings of CSEE, 26: 120-123, 2006. 
[17] http://www.pjm-cem.com/markets/energymarket/day-ahead.html.

[18] B.d. Liu, R.Q. Zhao, editor., Stochastic Programming and Fuzzy Programming, Tsinghua University Press, Beijing, 2001.

[19] M. Zhou, S.D. Sun, editor., SGenetic Algorithm Theory and Application, National Defense Industry Press, Beijing, 1999. 